\title{
Índice de sonolência diurna e correlação com a qualidade de vida: estudo com discentes de medicina e docentes médicos
}

\section{Daytime sleepiness index and correlation with quality of life: study with medical students and medical teachers}

Milena Nunes Alves de Sousa ${ }^{1,2^{*}}$, Marianny Macêdo de Lucena ${ }^{1}$, Yoshyara da Costa Anacleto Estrela $^{1}$, Joelly Holanda de Souza ${ }^{1}$, Élyta Palloma Rodrigues Brito ${ }^{1}$, Umberto Marinho de Lima Júnior $^{1}$, Tiago Bezerra de Sá de Sousa Nogueira ${ }^{1}$, Paulo Antônio Farias Lucena ${ }^{1}$

\section{RESUMO}

Objetivou-se avaliar o índice de sonolência diurna entre os estudantes de medicina e os docentes médicos correlacionando-o com sua qualidade de vida (QV). Foi conduzida pesquisa de campo, com caráter descritivo e abordagem quantitativa, junto a 200 alunos do curso de Medicina e 20 docentes médicos. Os dados foram obtidos a partir de dois questionários validados no Brasil: A Escala de Sonolência de Epworth (ESE) e o instrumento WHOQOL-BREF. Quanto à análise dos dados, além de estatística descritiva, utilizaram-se testes inferenciais de correlação de Pearson, Teste t de Studant e teste de quiquadrado de Pearson. Constatou-se que 69,1\% (152 participantes) da amostra apresentou sonolência diurna excessiva, houve um elevado índice indicado pela escala ESS-BR, cuja média foi de 11,48 pontos considerando os 2 grupos. Contudo, esse índice foi maior entre os estudantes de medicina do que entre os docentes médicos e afetou mais o sexo feminino. Os resultados obtidos alertam à realidade preocupante vivenciada pelos acadêmicos de medicina, e apontam a necessidade da adoção de medidas, além do PROCURAR, a fim de melhorar a QV desse grupo.

Palavras-Chave: Discentes; Docentes; Sonolência; Qualidade de Vida; Promoção da Saúde.

\begin{abstract}
The objective was to evaluate evaluate the daytime sleepiness index among medical students and medical teachers correlating it with their quality of life (QL). Was conducted using the field research, with a descriptive character and quantitative approach, with 200 medical students and 20 medical professors. The data were obtained from two questionnaires validated in Brazil: Epworth Sleepiness Scale (ESE) and WHOQOL-BREF instrument. As for data analysis, in addition to descriptive statistics, Pearson's correlation inferential tests, Studant's t-test and Pearson's chi-square test were used. It was found that 69.1\% (152 participants) of the sample presented excessive daytime sleepiness, there was a high index indicated by the ESS-BR scale, which showed an average of 11.48 points considering the 2 groups. However, this rate was higher among medical students comparing to medical teachers and affected more
\end{abstract}

\footnotetext{
${ }^{1}$ Centro Universitário de Patos (UNIFIP), Patos, Paraíba, Brasil.

* E-mail: milenanunes@ fiponline.edu.br

${ }^{2}$ Faculdade São Francisco da Paraíba (FASP), Cajazeiras, Paraíba, Brasil.
} 
the female sex. The results obtained alert to the reality experienced by medical students, and point out the necessity of adopting measures in order to improve the QL of this group.

Key words: Students; Teachers; Somnolence; Quality of life; Health Promotion.

\section{INTRODUÇÃO}

Existem inúmeros conceitos sobre o termo qualidade de vida tendo em vista sua subjetividade, no entanto, geralmente está correlacionado à saúde, referida pelo bemestar físico, funcional e mental. Além desses fatores, deve-se considerar a esfera das relações interpessoais como uma condição importante, pois, envolve elementos socioafetivos determinantes na vida dos indivíduos, como trabalho, família, amigos, ou outras circunstâncias do cotidiano (PEREIRA; TEIXEIRA; SANTOS, 2012).

Em virtude da relação intrínseca entre qualidade de vida e saúde, observa-se uma gama de causas plausíveis para uma redução no bem-estar do indivíduo, sendo uma delas a perturbação do ciclo sono-vigília. A alteração na quantidade ou na qualidade do sono tem repercussão direta nas atividades habituais do sujeito, que frequentemente apresenta sintomas como sonolência excessiva, fadiga e ainda comprometimentos cognitivos. Como consequência, observa-se a queda no desempenho em ambientes acadêmico, familiar ou de trabalho, causando grande impacto socioeconômico (PEREIRA; TEIXEIRA; SANTOS, 2012; AMERICAN ACADEMY OF SLEEP MEDICINE, 2014).

Esses impactos do sono não reparador no exercício de atividades da vigília podem ser observados na rotina de muitos estudantes de medicina e docentes médicos. No curso de medicina, há uma série de adversidades que justificam essa realidade: o turno integral, os estágios e horários fragmentados, a maior dedicação de tempo ao estudo e os inúmeros testes ou provas aos quais os alunos são submetidos. Enquanto os docentes médicos, muitas vezes, realizam plantões noturnos, tem uma elevada carga horária de trabalho e rotina irregular. Soma-se a esses fatores, a necessidade desses grupos conciliarem seus expedientes à vida social (FEODRIPPE; BRANDÃO; VALENTEL, 2013).

Seja pela alta exigência acadêmica ou laborativa, sejam pelos horários e rotinas irregulares, os acadêmicos de medicina e docentes médicos terminam alterando o seu 
padrão de sono a fim de atender às demandas necessárias, tornando-se, assim, mais propensos ao desenvolvimento da Sonolência Diurna Excessiva (MACHADO-DUQUE; ECHEVERRI CHABUR; MACHADO-ALBA, 2015).

Tendo em vista as consequências decorrentes da alteração do processo de sonovigília quanto à qualidade de vida e produtividade do indivíduo, este estudo objetivou avaliar o índice de sonolência diurna entre os estudantes de medicina e os docentes médicos correlacionando-o com sua qualidade de vida. Trata-se de um estudo pioneiro, o primeiro a avaliar essas duas variáveis e realizar a comparação entre acadêmicos de medicina e médicos.

\section{MATERIAL E MÉTODOS}

O trabalho foi realizado por meio da metodologia da pesquisa de campo, com caráter descritivo e abordagem quantitativa. A coleta dos dados foi realizada entre os meses de setembro e outubro de 2018 no campus Centro Universitário de Patos (UNIFIP), uma instituição educacional privada brasileira, com sede em Patos, localizada no sertão paraibano. A instituição oferece cursos de graduação e pósgraduação. Uma dos mais novos cursos ofertados é o de Medicina, que atualmente contempla até o $9^{\circ}$ período do processo de formação.

A amostra foi composta por 20 médicos docentes e por 200 acadêmicos do referido curso, sendo estes, estudantes do $1^{\circ}$ ao $8^{\circ}$ período que foram escolhidos de forma aleatória. $\mathrm{O}$ critério de inclusão utilizado foi à filiação discente/docente ao curso de medicina da UNIFIP. Foram excluídos os alunos que não estavam blocados e os alunos ou discentes em licença médica. Ressalta-se que a pesquisa não contemplou o $9^{\circ}$ período, pois possuem um padrão de rotina dessemelhante dos demais, uma vez que estão no internato.

Para a coleta dos dados foram utilizados dois questionários validados e autoaplicáveis: a Escala de Sonolência de Epworth (ESS-BR) (JOHNS, 1991) e o instrumento WHOQOL-BREF (FLECK et al., 2000).

A ESS-BR, desenvolvida pelo Dr. Murray Johns, foi utilizada para avaliar o índice de sonolência diurna. Foi traduzida e validada no Brasil em 2008, por Alessandra Naimaier Bertolazi. É composta pela pontuação de oito situações em que exista a chance de cochilar, que são: sentado e lendo, vendo televisão, sentado em lugar público 
e sem fazer atividades, sendo passageiro em trem, ônibus ou carro viajando 1 hora sem parar, estando deitado à tarde, sentado e conversando com alguém, sentado após o almoço, e passar muito tempo parado no trânsito quando dirige. A pontuação é indicada pelo entrevistado com valor 0 (sem probabilidade de cochilar), 1 (pequena probabilidade de cochilar), 2 (moderada probabilidade de cochilar) e 3 (alta probabilidade de cochilar). Após as respostas aos itens, somam-se esses valores: o somatório de 0 a 9 não indica sonolência diurna, e a soma de 10 a 24 indica sonolência diurna excessiva (JOHNS, 1991).

O segundo, formulado pela Organização Mundial de Saúde a partir do WHOQOL-100, foi utilizado para analisar a qualidade de vida. O WHOQOL-BREF foi validado no Brasil em 2002 e é constituído de 26 perguntas. As perguntas número 1 e 2 são sobre a qualidade de vida geral e além dessas, existem 24 facetas que compõem 4 domínios: físico (sono e repouso, energia e fadiga, dor e desconforto), psicológico (otimismo, memória, pensar, concentração, autoestima, aparência e imagem corporal), relações sociais (apoio social, relação pessoais e atividade sexual) e meio ambiente (segurança física e proteção, ambiente no lar, transporte, trânsito, ruído, clima, poluição, recursos financeiros e oportunidades de lazer). As respostas seguem uma escala de Likert que vai de 1 a 5, quanto maior a pontuação melhor a qualidade de vida (FLECK et al., 2000).

Os dados colhidos a partir dos dois instrumentos foram analisados no Programa Statistical Package for the Social Sciences (SPSS), versão 25.0. Além de estatística descritiva, utilizaram-se testes inferenciais de correlação de Pearson, Teste t de Studant e teste de qui-quadrado de Pearson. A significância estatística adotada foi de $p \leq 0,05$.

A pesquisa foi iniciada após a aprovação do Comitê de Ética em Pesquisa (CEP) do UNIFIP, CAAE: 95791318.8.0000.5181/ Número do Parecer: 2.872.206/2018.

\section{RESULTADOS}

A amostra foi composta por 220 participantes. Desse total, 200 eram acadêmicos de medicina do $1^{\circ}$ ao $8^{\circ}$ período, sendo 130 do sexo feminino e 70 do sexo masculino, e 20 eram docentes médicos, sendo 5 do sexo feminino e 15 do sexo masculino.

Após a análise das pontuações feitas na ESS-BR, evidenciou-se que 69,1\% $(\mathrm{n}=152)$ participantes da amostra apresenta sonolência diurna excessiva. Quanto ao 
sexo, 135 participantes $(61,4 \%$ da amostra) eram do sexo feminino e 78,5\% desse total (106) apresentaram SDE, enquanto $85(38,6 \%)$ eram do sexo masculino e 54,1\% desse total $(n=46)$ apresentaram SDE. Quanto à categoria profissional, 170 participantes eram acadêmicos de medicina e $72 \%$ desse total $(n=144)$ apresentou SDE, enquanto 20 eram docentes médicos, dos quais 40\% (n=8) apresentou SDE (valor de $\mathrm{p}<0,01$ ).

Ao avaliar as médias das pontuações do WHOQOL-BREF e da ESS-BR, descritas na tabela 1, viu-se que a média de qualidade de vida mais elevada foi no domínio social e a mais no físico, e que a SDE apresentou-se bastante significativa (a média das pontuações foi de 11, para ESS-BR valores entre 10-24 indicam SDE).

Tabela 1 - Descrição das médias de idade, de sonolência e de qualidade de vida amostra

\begin{tabular}{cccccc}
\hline & Média & $\begin{array}{c}\text { Desvio } \\
\text { padrão }\end{array}$ & Mediana & Mínimo & Máximo \\
\hline Idade & 24,08 & 5,93 & 22,00 & 17 & 46 \\
QV física & 60,79 & 15,09 & 60,71 & 17,86 & 100,00 \\
QV psicológico & 61,98 & 15,80 & 62,50 & 4,17 & 95,83 \\
QV social & 67,00 & 19,85 & 66,66 & 0,00 & 100,00 \\
QV ambiental & 63,32 & 13,44 & 62,50 & 25,00 & 100,00 \\
QV geral & 62,72 & 20,02 & 62,50 & 0,00 & 100,00 \\
Sonolência & 11,48 & 3,95 & 11 & 2,00 & 24,00 \\
\hline
\end{tabular}

Fonte: Pesquisa direta, 2018.

A tabela 2 mostra que os docentes médicos apresentaram maiores médias de qualidade de vida nos domínios físico, psicológico e ambiental em relação aos acadêmicos. Estas diferenças foram estatisticamente significativas.

Tabela 2 - Comparação de qualidade de vida entre categorias (Docentes X discentes)

\begin{tabular}{lccccc}
\hline & QV física & $\begin{array}{c}\text { QV } \\
\text { psicológico }\end{array}$ & QV social & $\begin{array}{c}\text { QV } \\
\text { ambiental }\end{array}$ & QV geral \\
\hline Acadêmicos de medicina & $\underline{59,28}$ & $\underline{60,70}$ & 66,41 & $\underline{62,59}$ & 62,37 \\
$\begin{array}{l}\text { Média } \\
\text { Desvio padrão }\end{array}$ & 14,52 & 15,67 & 19,67 & 13,05 & 19,93 \\
$\begin{array}{l}\text { Docentes médicos } \\
\text { Média }\end{array}$ & $\underline{75,89}$ & $\underline{74,79}$ & 72,91 & $\underline{70,62}$ & 66,25 \\
Desvio padrão & 12,41 & 10,77 & 21,09 & 15,35 & 21,11 \\
\hline p-valor & $\mathbf{0 , 0 1}$ & $\mathbf{0 , 0 1}$ & 0,16 & $\mathbf{0 , 0 1}$ & 0,41 \\
\hline
\end{tabular}

Fonte: Pesquisa direta, 2018. 
A tabela 3 mostra que o sexo masculino apresentou maiores médias de qualidade de vida nos domínios físicos, psicológicos e ambiental. Estes resultados foram estatisticamente significativos.

Tabela 3 - Comparação de qualidade de vida entre sexo

\begin{tabular}{lccccc}
\hline & QV física & $\begin{array}{c}\text { QV } \\
\text { psicológico }\end{array}$ & QV social & $\begin{array}{c}\text { QV } \\
\text { ambiental }\end{array}$ & QV geral \\
\hline $\begin{array}{l}\text { Feminino } \\
\text { Média }\end{array}$ & 57,40 & 59,75 & 66,72 & 61,57 & 61,20 \\
$\begin{array}{l}\text { Desvio padrão } \\
\text { Masculino }\end{array}$ & 13,78 & 14,00 & 18,64 & 12,22 & 19,98 \\
Média & & & & & \\
Desvio padrão & $\mathbf{6 6 , 1 7}$ & $\mathbf{6 5 , 5 3}$ & 67,45 & $\mathbf{6 6 , 1 0}$ & 65,14 \\
\hline p-valor & 15,59 & 17,82 & 21,73 & 14,82 & 19,96 \\
\hline
\end{tabular}

Fonte: Pesquisa direta, 2018.

A tabela 4 mostra que os domínios físico, psicológico e ambiental apresentaram correlação significativa e negativa com a sonolência.

Tabela 4 - Correlação entre qualidade de vida e sonolência

\begin{tabular}{lll}
\hline & & Sonolência \\
\hline QV física & Correlação & $-0,26$ \\
& Sig. & $\mathbf{0 , 0 1}$ \\
\hline QV psicológico & Correlação & $-0,21$ \\
\hline QV social & Sig. & $\mathbf{0 , 0 1}$ \\
\hline QV ambiental & Correlação & $-0,05$ \\
& Sig. & 0,43 \\
\hline QV geral & Correlação & $-0,16$ \\
& Sig. & $\mathbf{0 , 0 1}$ \\
\hline
\end{tabular}

Fonte: Pesquisa direta, 2018.

A tabela 5 mostra correlação significativa e negativa da qualidade de vida nos domínios físico, psicológico e ambiental com a sonolência para os acadêmicos de medicina. Os docentes apresentaram correlação positiva e estatisticamente significativa do domínio social com a sonolência. 
Tabela 5 - Correlação entre qualidade de vida e sonolência, em função da categoria profissional.

\begin{tabular}{llcc}
\hline & & \multicolumn{2}{c}{ Categoria profissional } \\
\hline & & $\begin{array}{c}\text { Acadêmicos de } \\
\text { medicina }\end{array}$ & Docentes médicos \\
\hline QV física & Correlação & $-0,22$ & $-0,24$ \\
& Sig. & $\mathbf{0 , 0 1}$ & 0,15 \\
\hline QV psicológico & Correlação & $-0,17$ & $-0,11$ \\
& Sig. & $\mathbf{0 , 0 1}$ & 0,31 \\
\hline QV social & Correlação & $-0,07$ & 0,33 \\
& Sig. & 0,14 & $\mathbf{0 , 0 5}$ \\
\hline QV ambiental & Correlação & $-0,17$ & 0,13 \\
& Sig. & $\mathbf{0 , 0 1}$ & 0,28 \\
\hline & Correlação & $-0,05$ & $-0,14$ \\
& Sig. & 0,21 & 0,26 \\
\hline
\end{tabular}

Fonte: Pesquisa direta, 2018.

\section{DISCUSSÃO}

O sono é um mecanismo fisiológico fundamental ao equilíbrio biopsicossocial do ser humano, pois promove uma série de alterações fisiológicas, responsáveis pela regulação das funções corporais e psíquicas do organismo. É caracterizado pelo seu aspecto cíclico e constituído por fases distintas de acordo com três variáveis fisiológicas, que são: o eletroencefalograma (EEG), o eletro-oculograma (EOG) e o eletromiograma (EMG). De acordo com esses elementos, pode-se dividi-lo em dois estágios: fase Non-Rapid Eye Movement (NREM), que por sua vez é subdividida em N1, N2 e N3, e a fase Rapid Eye Movement (REM) (OBAL JÚNIOR; KRUEGER, 2004; RODRIGUES et al., 2012; NEVES et al., 2013).

Algumas das funções do sono são: permitir a manutenção de processos promotores da vigília, promover a conservação de energia e viabilizar os processos anabólicos, havendo secreção de hormonal (GH, TSH, ACTH e prolactina), atuar na termorregulação central e na limpeza de toxinas que são acumuladas no cérebro durante a vigília, estimular a liberação de citocinas, promover a plasticidade do cérebro e a consolidação da memória, e atuar na regulação de processos metabólicos cujos distúrbios podem resultar em patologias como diabetes, hipertensão arterial, obesidade, 
doença coronariana, depressão, ansiedade e insônia (OBAL JÚNIOR; KRUEGER, 2004; VERPES, 2004; PAIVA; PENZEL, 2011; NEVES et al., 2013).

Por influenciar todos esses mecanismos, um sono de boa qualidade permite a manutenção da vigília e o desempenho eficaz das atividades durante o dia. Dessa forma, indivíduos que têm privação do sono ou um sono de má qualidade apresentam maior sonolência diurna, menor desempenho, e menor qualidade de vida (NEVES et al., 2013).

Seja pela alta exigência acadêmica ou laborativa, sejam pelos horários e rotinas irregulares, os acadêmicos de medicina e docentes médicos terminam alterando o seu padrão de sono a fim de atender às demandas necessárias, sujeitando-se a ficarem mais propensos ao desenvolvimento da Sonolência Diurna Excessiva (SDE). Fato corroborado pelo índice de sonolência diurna na pesquisa, em que $72 \%$ dos acadêmicos e $40 \%$ dos docentes médicos avaliados apresentaram SDE (10 ou mais pontos na escala), com média de pontuação de 11,48 pontos. Proporcionalmente, observou-se maior frequência da SDE no sexo feminino (78,5\% de 135) e em acadêmicos de medicina (72\% de 200).

Comparando os resultados obtidos com trabalhos semelhantes, constata-se que podem ocorrer variações nesses índices conforme se modifica a instituição de ensino, a realidade em que se está inserida e o método de aprendizagem adotado. Em uma pesquisa realizada com estudantes de medicina na Faculdade de Medicina da Universidade de Coimbra, a qual utilizou como instrumento o ESS-BR, o mesmo usado nesta pesquisa, viu-se que a prevalência de SDE na amostra foi de $31,4 \%$, e a média na pontuação da escala foi 7.85 , sem diferenças significativas em relação ao sexo (ROSMANINHO, 2011).

Contudo, outro estudo realizado com estudantes de medicina da Faculdade de Ciências Médicas e da Saúde da Pontifícia Universidade Católica de São Paulo, que também utilizou o referido instrumento de coleta de dados, apontou sonolência excessiva em 36,31\% dos estudantes, com maior predominância no sexo feminino (MORAES et al., 2013). E em um terceiro estudo com acadêmicos de medicina de uma instituição privada de Curitiba, utilizando a mesma escala, observou-se que 49,1\% (84 estudantes) da amostra apresentou escores patológicos de SDE, cuja média foi de 10,6 (KLOSTER et al., 2013). 
Nota-se que os números obtidos na pesquisa com os acadêmicos de medicina do UNIFIP, em relação à sonolência diurna excessiva, foram mais elevados do que aqueles verificados nos estudos anteriormente citados. Embora se reflita para o fato de que análises de caráter somente quantitativo não devam ser comparadas entre si sem que haja uma contextualização adequada da realidade vivida por cada instituição.

O curso é recente e atualmente está com o $9^{\circ}$ período em andamento. Adota, predominantemente, o método de aprendizagem Problem Based Learning (PBL), uma metodologia ativa, centrada no aluno, considerada muito eficaz e mais exaustiva do que o método de ensino tradicional (MIRANDA JUNIOR, 2016).

Postula-se que esse elevado índice de sonolência diurna é um reflexo da sobrecarga gerada pela vida acadêmica, pois os estudantes precisam conciliar horários irregulares, estágios, produção científica e tempo para estudo, são submetidos a avaliações teóricas semanais ou quinzenais, e ao final de cada módulo, além da teoria, é feita também a avaliação de habilidades práticas. Existem ainda atividades extracurriculares como projetos de extensão, matérias optativas e ligas. Em virtude da rotina acadêmica intensa, muitas vezes, é necessário abdicar de horas de sono para atender a todas as demandas de estudo, ou em decorrência do elevado nível de estresse há prejuízo na qualidade do sono, levando à sonolência diurna excessiva. Sabendo das circunstâncias às quais os alunos estão submetidos, o UNIFIP dispõe de um Programa de Apoio Psicossocial ao Estudante de Medicina (PRO-CURAR), que buscar promover a saúde mental, individual e coletiva dessa comunidade acadêmica mediante, por exemplo, de escutas individuais junto à duas psicólogas e um psiquiatra, docentes da instituição.

Em relação aos docentes médicos, houve uma dificuldade na distribuição e recolhimento dos questionários. Contudo, do total analisado, observou-se que a sonolência diurna nesse grupo é de $40 \%$, menor que a dos discentes, embora a rotina dessa classe também seja laboriosa.

A SDE, identificada nesses grupos, é caracterizada por uma compulsão subjetiva para dormir, que não varia de acordo com o dia-a-dia, nem com o ritmo circadiano como se é esperado. Esse quadro gera déficit cognitivo, lapsos de memória, perda da capacidade de concentração, cansaço, fadiga e desmotivação, o que pode provocar diminuição da produtividade acadêmica ou profissional. Além disso, por aumentar os 
níveis estresse e irritabilidade, pode interferir também no estado mental e na qualidade das relações sociais estabelecidas pelos indivíduos (ALMOJALI et al., 2017).

Em concordância ao fato exposto acima, observou-se a inversão proporcional entre sonolência diurna excessiva e qualidade de vida. $\mathrm{O}$ estudo revelou uma correlação significativa e negativa nos domínios físico, psicológico e ambiental com a sonolência para os acadêmicos de medicina, e uma correlação significativa e negativa do domínio social com a sonolência para os docentes médicos.

Nota-se que os discentes apresentaram um maior índice de SDE do que os docentes, sendo mais prevalente no sexo feminino. Houve, por conseguinte, um maior prejuízo à qualidade de vida nesse grupo, principalmente no que se refere à QV física (relacionada ao sono e repouso, energia e fadiga, dor e desconforto), psicológica (relacionada a otimismo, memória, pensar, concentração, autoestima, aparência e imagem corporal) e ambiental (relacionada à segurança física e proteção, ambiente no lar, transporte, trânsito, ruído, clima, poluição, recursos financeiros e oportunidades de lazer). Desses três domínios o que obteve pior média foi o de QV física $(59,28)$, seguido pelo psicológico $(60,70)$ e ambiental $(62,59)$. A esfera mais bem avaliada foi a de relações sociais com média de 66,41 pontos, que está relacionada ao apoio social, relações pessoais e atividade sexual. Quanto à qualidade de vida geral, a média entre os estudantes foi de 62,37 pontos.

Associado ao maior índice de sonolência no sexo feminino, viu-se também que as médias de qualidade de vida referentes aos domínios físico, psicológico e ambiental foram inferiores àquelas verificadas no sexo masculino, havendo significância estatística.

Em uma pesquisa que abordou a qualidade de vida em estudantes de medicina da FIP, realizada em 2016, a QV social também foi o domínio com maior média $(69,2)$. Mas, esse mesmo estudo revelou que a segunda esfera melhor pontuada foi a de QV psicológica $(65,36)$, contrastando com os resultados aqui expostos. A QV geral demonstrou escore de 68,38 pontos (PEREIRA et al., 2017). Comparando as médias de qualidade de vida obtidas nos dois estudos, observou-se que houve uma queda na qualidade de vida geral e em todos os domínios (social, psicológico, físico e ambiental) nesse período. Deve-se levar em consideração o aumento da amostra, pois, aquela do ano de 2016 contava com estudantes do primeiro ao quinto período, enquanto esta abrange do primeiro ao oitavo. 
Em outras diversas pesquisas sobre a qualidade de vida em discentes de medicina de outras instituições, que utilizaram o WHOQOL-BREF, o domínio psicológico foi o que recebeu o mais baixo escore na avaliação (HASSED et al., 2009; ALVES et al., 2010; RAMOS-DIAS et al., 2010; BAMPI et al., 2013;). O dado é preocupante, haja vista estudos apontarem os transtornos psicológicos com estimativas e evidências crescentes no grupo (GUEDES et al., 2017; ESTRELA et al., 2018; SOUSA et al., 2020; SOUSA et al., 2021).

De acordo com um trabalho que visou analisar a relação entre qualidade de vida e problemas de saúde mental em estudantes de medicina do UNIFIP, foi evidenciada a relação inversa entre essas duas variáveis. Ou seja, aqueles que sofriam com algum problema mental, como estresse, Síndrome de Burnout ou depressão tiveram sua qualidade de vida significativamente afetada, refletindo diretamente na forma do cuidado, no desenvolvimento das habilidades práticas e em seu desempenho acadêmico (RIBEIRO et al., 2018). Para entender a relevância dessa problemática, vale destacar que $89,1 \%$ da amostra de estudantes do mesmo curso e instituição de ensino, analisados em estudo anterior, relataram que às vezes necessitam de tratamento psiquiátrico (ALMOJALI et al., 2017).

Quanto aos docentes médicos, apresentaram prejuízo significativo apenas da QV social quando comparados aos acadêmicos. No que se referem à esfera física, psicológica e ambiental, as médias foram superiores àquelas verificadas nos estudantes.

Em uma pesquisa em que foram avaliados 1100 médicos paraibanos constatouse que a maioria dos profissionais apresentou uma alta qualidade de vida. Supõe-se que isso está associado à estabilidade financeira adquirida, devido à boa remuneração e ao baixo custo de vida, permitindo um maior conforto. Foi revelada a existência de correlação negativa entre QV e o número de plantões realizados ou carga horária intensa de trabalho, pois essas situações podem causar um impacto negativo em sua saúde física e mental. Viu-se ainda que quanto maior a idade do médico, menor a QV física e maior a QV psicológica. Provavelmente, em decorrência do prejuízo à saúde física, esses profissionais acabam reduzindo as longas jornadas de trabalho, resultando em maior bem-estar psicológico (AZEVEDO; MATHIAS, 2017). Um segundo trabalho, com médicos ortopedistas, obteve resultados semelhantes, evidenciando altos escores nos domínios do WHOQOL-100, exceto no físico. Ainda assim, os profissionais têm a percepção de uma boa qualidade de vida (MELLO; SOUZA, 2013). 
Já em outro estudo, o qual avaliou a qualidade de vida de 62 médicos da atenção primária de Roraima, estado da região Norte do Brasil, obteve escores elevados quanto à QV física, psicológica e social, porém, foram identificados fatores estressores relacionados à insatisfação com o ambiente e as condições de trabalho, que interferiram negativamente na qualidade de vida desses profissionais (OLIVARES; BONITO; SILVA, 2015).

Portanto, é fundamental que sejam adotadas estratégias de enfrentamento, pois já se reconhece que vários aspectos de vida de estudantes e profissionais podem ser afetados, tais como impactar negativamente sobre a qualidade de vida e corroborar com o desfecho da síndrome de Burnout (RIBEIRO; SOUSA, 2020).

Por fim, como limitações identificadas no estudo destaca-se a dificuldade da aplicação dos questionários aos docentes, apenas 20 dos 45 participaram da pesquisa, ademais, também pela escassez de trabalhos que abordam a qualidade de vida em médicos ou docentes médicos para realizar a comparação dos resultados.

\section{CONCLUSÃO}

Verificou-se que há um elevado índice de sonolência diurna excessiva indicada pela escala ESS-BR, cuja média foi de 11,48 pontos considerando os dois grupos. Contudo, o índice foi maior entre os estudantes de medicina do que entre os docentes médicos da Instituição de Ensino Superior analisada, refletindo assim nos níveis de qualidade de vida, em que mais uma vez os acadêmicos apresentaram escores, no geral, menores do que os docentes.

Infere-se que o suporte psicológico oferecido pela instituição interfere positivamente na saúde mental dos estudantes, pois, ao contrário do que foi visto em outros estudos, a QV psicológica desse grupo não foi a mais prejudicada, e sim a QV física.

Os resultados obtidos nesse estudo alertam a realidade preocupante vivenciada pelos acadêmicos de medicina e apontam a necessidade da adoção de medidas, além do PRO-CURAR, a fim de melhorar a qualidade de vida desse grupo na medida do possível. Como sugestões, dentro as ações que podem ser incentivadas destacam-se as de valorização da vida, do estímulo a atividades recreativas, associativas e de lazer, além de uma maior participação discente nos processos de gestão do curso. Sugere-se ainda, a mudança no horário de início das aulas de 07:00 horas para as 08:00 horas da manhã. 


\section{REFERÊNCIAS}

ALMOJALI, Abdullah I. et al. The prevalence and association of stress with sleep quality among medical students. Journal of epidemiology and global health, v. 7, n. 3, p. 169-174, 2017.

ALVES, João Guilherme Bezerra et al. Qualidade de vida em estudantes de Medicina no início e final do curso: avaliação pelo Whoqol-bref. Revista Brasileira de

Educação Médica, v. 34, p. 91-96, 2010.

AMERICAN ACADEMY OF SLEEP MEDICINE. International classification of sleep disorders. 3. ed. Westchester: American Academy of Sleep Medicine, 2014.

AZEVEDO, Walter Fernandes de; MATHIAS, Lígia Andrade da Silva Telles. Work addiction and quality of life: a study with physicians. Einstein (São Paulo), v. 15, p. 130-135, 2017.

BAMPI, Luciana Neves da Silva et al. Qualidade de vida de estudantes de Medicina da Universidade de Brasília. Revista Brasileira de Educação Médica, v. 37, p. 217-225, 2013.

ESTRELA, Yoshyara da Costa Anacleto et al. Estresse e correlatos com características de saúde e sociodemográficas de estudantes de medicina. CES Medicina, v. 32, n. 3, p. 215-225, 2018.

FEODRIPPE, André Luiz Oliveira; BRANDÃO, Maria Carolina da Fonseca;

VALENTE, Tânia Cristina de Oliveira. Qualidade de vida de estudantes de Medicina: uma revisão. Revista Brasileira de Educação Médica, v. 37, n. 3, p. 418-428, 2013.

FLECK, Marcelo et al. Aplicação da versão em português do instrumento abreviado de avaliação da qualidade de vida" WHOQOL-bref". Revista de saúde pública, v. 34, p. 178-183, 2000.

GUEDES, Anderson Ferreira et al. Fatores de risco para o estresse entre estudantes da área de saúde. Revista Coopex, v. 8, p. 1-10, 2017.

HASSED, Craig et al. Enhancing the health of medical students: outcomes of an integrated mindfulness and lifestyle program. Advances in health sciences education, v. 14, n. 3, p. 387-398, 2009.

JOHNS, Murray W. A new method for measuring daytime sleepiness: the Epworth sleepiness scale. sleep, v. 14, n. 6, p. 540-545, 1991.

KLOSTER, Maria Cristina et al. Sonolência diurna e habilidades sociais em estudantes de medicina. Revista Brasileira de Educação Médica, v. 37, p. 103-109, 2013. 
MACHADO-DUQUE, Manuel Enrique; ECHEVERRI CHABUR, Jorge Enrique; MACHADO-ALBA, Jorge Enrique. Excessive daytime sleepiness, poor quality sleep, and low academic performance in medical students. Revista colombiana de psiquiatria, v. 44, n. 3, p. 137-142, 2015.

MELLO, Marcelo Henrique de; SOUZA, José Carlos. Qualidade de vida dos médicos ortopedistas do Mato Grosso do Sul. Revista Brasileira de Ortopedia, v. 48, p. 92-99, 2013.

MIRANDA JUNIOR, Ubirajara José Picanço de. Problem Based Learning como metodologia inovadora no ensino de graduação em saúde. Revista Portuguesa de Medicina Geral e Familiar, v. 32, n. 1, p. 12-3, 2016.

MORAES, Caio A. T. et al. Qualidade de sono em estudantes de medicina do método de aprendizado baseado em problemas. Medicina (Ribeirão Preto), v. 46, n. 4, p. 389397, 2013.

NEVES, Gisele S. Moura et al. Transtornos do sono: visão geral. Rev Bras Neurol, v. 49, n. 2, p. 57-71, 2013.

OBAL JÚNIOR, Ferenc; KRUEGER, James M. GHRH and sleep. Sleep medicine reviews, v. 8, n. 5, p. 367-377, 2004.

OLIVARES, Alberto; BONITO, Jorge; SILVA, Rozinaldo. Qualidade de vida no trabalho dos médicos da atenção básica no estado de Roraima (Brasil). Psicol Saúde Doenças, v. 16, n. 1, p. 100-111, 2015.

PAIVA, T.; PENZEL, T. Características Básicas do sono. Centro de Medicina do Sono - Manual prático. Lidel, 2011.

PEREIRA, Érico Felden; TEIXEIRA, Clarissa Stefani; SANTOS, Anderlei dos. Qualidade de vida: abordagens, conceitos e avaliação. Revista brasileira de educação física e esporte, v. 26, p. 241-250, 2012.

PEREIRA, F. E. L. et al. Correlatos da qualidade de vida com características de saúde e demográficas de estudantes de medicina. Rev Bras Qual Vida, v. 9, p. 247-60, 2017.

RAMOS-DIAS, João Carlos et al. Qualidade de vida em cem alunos do curso de Medicina de Sorocaba-PUC/SP. Revista Brasileira de Educação Médica, v. 34, p. 116-123, 2010.

RIBEIRO, R. C. et al. Relação da qualidade de vida com problemas de saúde mental em universitários de medicina. Rev Bras Qual Vida, v. 10, p. e7646, 2018.

RIBEIRO, Rafaella do Carmo; SOUSA, Milena Nunes Alves de. Relationship between coping strategies, dimensions of burnout syndrome and life quality in medical students. Rev Enferm UFPI, v. 9, n. 1, 2020.

RODRIGUES, Maria Helena et al. Polissonografia: aspectos técnicos e clínicos. Rev. bras. neurol, 2012. 
ROSMANINHO, J. M. C. Sonolência diurna em estudantes de medicina. 2011. Tese (Doutorado) - Faculdade de Medicina da Universidade de Coimbra. Coimbra, 2011.

SOUSA, Milena Nunes Alves et al. Correlatos das dimensões de Burnout com características de saúde e demográficas de estudantes de medicina. CES Medicina, v. 34 , n. 1, p. 27-39, 2020.

SOUSA, Milena Nunes Alves et al. Transtornos emocionais em estudantes de medicina. Brazilian Journal of Health Review, v. 4, n. 1, p. 2894-2905, 2021.

Recebido em: 03/11/2021

Aprovado em: 20/11/2021

Publicado em: 30/11/2021 